\title{
Fabrication of nano-biocomposite PLA incorporated cellulose nanocrystal and reduced graphene oxide for medical application
}

\author{
Nidhi Pal and Kaushik Pal* \\ Department of Mechanical and Industrial Engineering, \\ Indian Institute of Technology Roorkee-247667, India \\ *Email:pl_kshk@yahoo.co.in
}

PLA has been extensively used in packaging applications because of its biodegradability, flexibility and low in cost. Cellulose nanocrystal having excellent mechanical strength, barrier properties and reduced graphene oxide because of antibacterial response both of them can be used as a promising nanofiller [1].In our present work, Poly lactic acid (PLA), incorporated with cellulose nanocrystal (CNC) and reduced graphene oxide (rGO) a nano-biocomposite film was fabricated through solvent casting method. The nano-biocomposite films fabricated have applications in the field of medical as well as in packaging sector.

In the synthesis of the nano-biocomposites, the $\mathrm{CNC}$ was acid hydrolyzed with $64 \%$ sulphuric acid and it was then centrifuged. The precipitate obtained was then sonicated using tip sonicator with $60 \%$ output. After that, the precipitate obtained was freeze dried using lyophillizer. The rGO was synthesized by treating graphene oxide with hydrazine hydrate. The GO was dissolved into deionized water and then stirred at $100{ }^{\circ} \mathrm{C}$ using reflux condenser. The precipitate was then washed using methanol and deionized water. The PLA pellets was first dissolved in chloroform and then the $\mathrm{CNC}$ and rGO dissolved in chloroform was added into the PLA solution. After that, the solution was cast onto a petri dish and the film was peeled off.

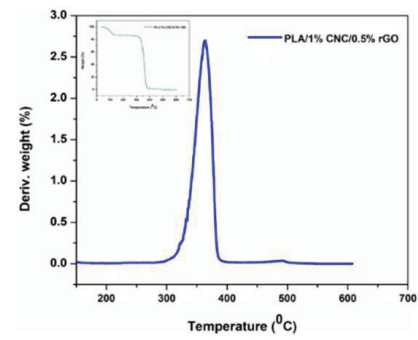

Figure 1: DTG and TGA curve of nanobiocomposite

The film thickness was ca. $70 \mu \mathrm{m}$. The film obtained was then characterized using different characterization techniques. One of the characterization technique is Thermo Gravimetric Analysis (TGA). Figure 1 shows the DTG and TGA curve of the PLA-CNC-rGO nanocomposite film. The maximum thermal degradation temperature can be calculated by DTG curve and the TGA curve helped in finding the onset temperature [2]. The film synthesized was found to be thermally stable with increase in the degradation and onset temperature of PLA. Figure 2 shows the MTT assay test for the nano-biocomposite film. From the figure it can be investigated that the film has low toxicity.

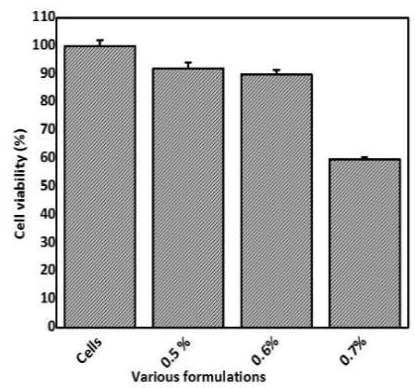

Figure 2: Cytotoxicity of nano-biocomposite

In the present study, the PLA incorporated film with $\mathrm{CNC}$ and $\mathrm{rGO}$ was fabricated using solvent casting method. The $\mathrm{CNC}$ and $\mathrm{rGO}$ was successfully incorporated into PLA matrix. The nano-biocomposite was found to be having thermal stability with increase in degradation and onset temperature. The cytocompatibility test showed that the film has having low toxicity .The film obtained can be successfully used in packaging as well as in medical applications.

\section{References}

1. B.W. Chieng, N. A. Ibrahim, W. M. Z. W. Yunus, M. Z. Hussein, Polymers 6 (2014) 93-104.

2. C. Zhang, L. Wang; T. Zhai, X. Wang, Y. LihSheng Turng, J. Mech Behav Biomed Mater 53(2016) 403-413. 